\title{
Modulatory mechanisms controlling the NLRP3 inflammasome in inflammation: recent developments
}

Haneklaus M, O'Neill LA, Coll RC, Modulatory mechanisms controlling the NLRP3 inflammasome in inflammation: recent developments., Current opinion in immunology, 25, (1), 2013, p40-45

The protein NLRP3 has emerged as a central regulator in the inflammatory process, being implicated directly in hereditary cryopyrinopathies, and indirectly in diseases such as gout, Type 2 diabetes and atherosclerosis. NLRP3 is an important regulator of caspase- 1 , the enzyme that processes the immature form of IL- $1 \beta$ into the active protein. The control of NLRP3 has therefore become a focus of research with evidence for redox regulation, ubiquitination and regulation by miRNA-223, kinases and calcium all emerging as controllers of NLRP3. As our knowledge expands the prospect for precise pharmacological targeting of NLRP3 will improve and could lead to substantial clinical utility.

\section{Introduction}

The pro-inflammatory cytokine IL- $1 \beta$ is the most studied of all cytokines because of its central role in the inflammatory process. IL-1 $\beta$ has also been implicated in many diseases, most recently in metabolic diseases such as gout, Type 2 diabetes (T2D) and atherosclerosis [1]. One reason for the recent focus on these particular diseases is the discovery of NLRP3, the key protein in the mechanism by which IL-1 $\beta$ production is regulated in macrophages [2]. IL-1 $\beta$ is initially made as a pro-form and is processed to the mature cytokine by caspase- 1 , which occurs in a complex with NLRP3 and ASC in a multi-protein complex termed the inflammasome [3]. The major function of NLRP3 is to sense phagocytosed material, and relay the signal to caspase-1 [4]. Diseases such as gout, T2D and atherosclerosis can be considered 'particle diseases' since there are roles for insoluble particles in their pathogenesis. Specifically, gout involves crystals of uric acid [5], T2D the amyloid protein Islet Amyloid Polypeptide [6] and atherosclerosis cholesterol crystals [7]. All of these have been shown to be phagocytosed by macrophages, activating NLRP3. NLRP3 has also been shown to be mutated in human in familial fevers termed cryopyrinopathies [8], and this discovery has allowed NLRP3 to move to centre stage as the key regulator of caspase- 1 and IL-1 $\beta$ in human and generate considerable interest from the pharma sector [9•]. Attention has moved to the regulation of NLRP3, since dysregulation can obviously be seen as a potential cause of disease pathogenesis. Here we discuss recent insights into the modulation of NLRP3. Roles of reactive oxygen species (ROS), control by microRNA (miRNA), ubiquitination, phosphorylation and calcium are discussed. We also discuss the prospect of targeting NLRP3 with small molecule inhibitors, which could hold great promise clinically. 
A major recent area of interest has been the role of ROS in NLRP3 regulation but there is much controversy here and precisely how ROS might regulate NLRP3 is still unknown. An often-repeated finding is that antioxidants are potent inhibitors of NLRP3-dependent IL-1 $\beta$ production, which prompted a great deal of research on the role of ROS and oxidative stress in NLRP3 activation. Even though an involvement of redox signalling is generally accepted, many conflicting results have been reported. For example, ROS originating from the NADPH oxidase complex were originally found to be important [10]. However, macrophages from mice or humans with genetically defective NADPH oxidase had normal NLRP3 activation [11 and 12]. Subsequently, mitochondrial ROS were put forward as being essential for inflammasome activation, a finding that is supported by the co-localization of NLRP3 inflammasomes with mitochondria in the perinuclear space upon activation [13•].

Functionally, ROS were proposed to be exclusively involved in the 'priming' step of NLRP3 activation. Initially, ROS induction by TLRs was shown to be required for NFKB activation, which is the traditional priming signal and induces transcription of NLRP3 and pro-IL-1 $\beta$ [14]. Recently, a novel priming mechanism has been put forward, which results in the post-translational activation of NLRP3 by de-ubiquitination and also requires TLR-induced ROS, as described later [15••]. Despite these findings, two redox-related factors have also been suggested to be direct NLRP3-activating ligands. Firstly, thioredoxin-interacting protein (TXNIP) has been shown to dissociate from thioredoxin (TRX) upon oxidative stress, which allows it to directly bind to NLRP3 [16]. However, the involvement of TXNIP in NLRP3 activation in BMDMs could not be confirmed in other studies [6]. Secondly, Shimada et al. recently suggested oxidized mitochondrial DNA as a NLRP3 ligand [ 17••]. They find that upon inflammasome activation or during apoptosis, dysfunctional mitochondria can release oxidized DNA into the cytosol. In the presence of a priming stimulus, this leads to IL-1 $\beta$ production. This also ties in the intrinsic apoptotic pathway with inflammasome signalling, which share striking similarities at the level of the mitochondria ( Figure 1). 
Figure 1.

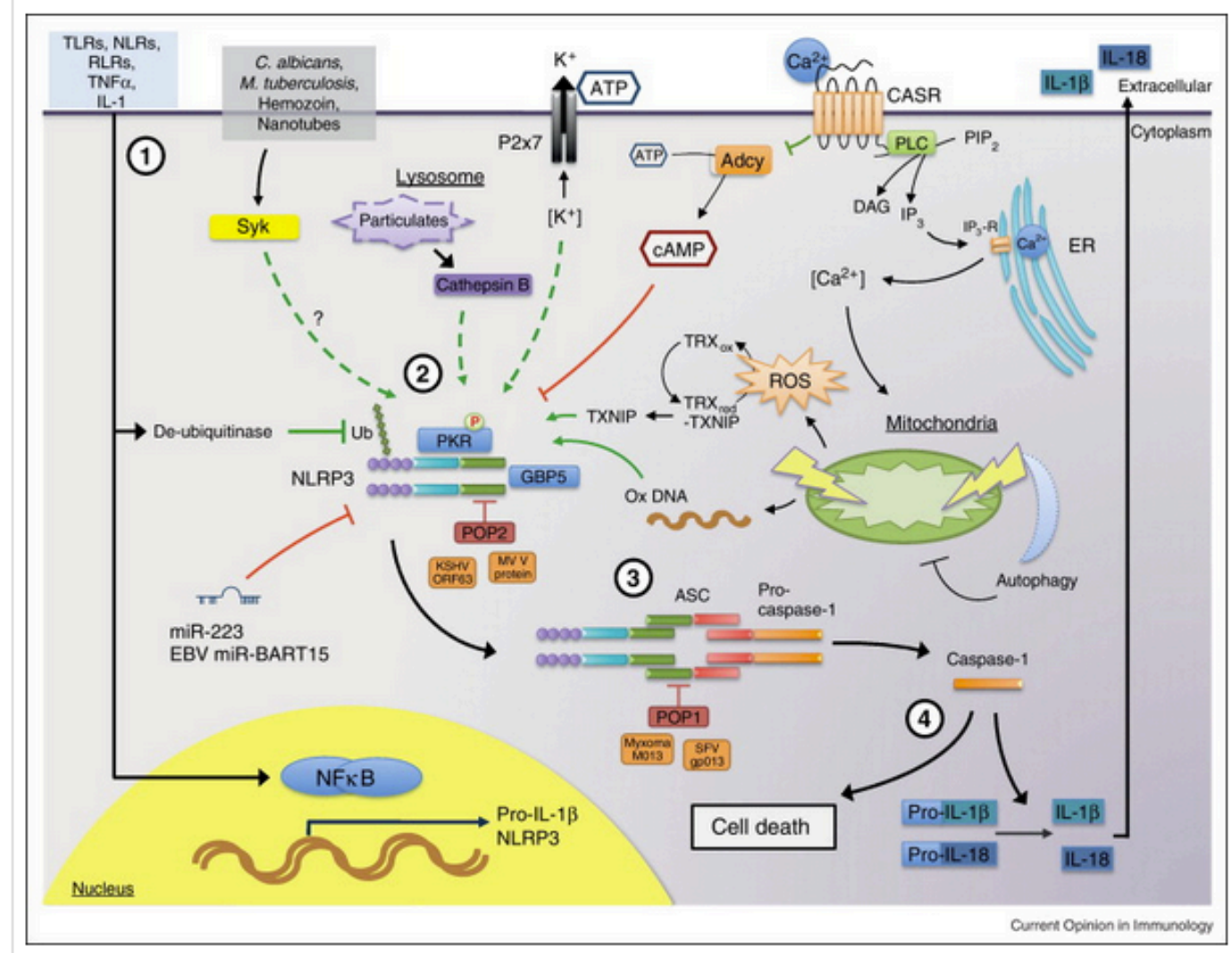

Current view of NLRP3 inflammasome activation. The initiating step in NLRP3 activation is a 'priming' signal (1) by pattern recognition receptors, most commonly Toll-like receptors that activate transcription of NLRP3 and pro-IL-1 $\beta$ through NFKB. At the same time, NLRP3 is activated non-transcriptionally by deubiquitination. NLRP3 levels can further be regulated by miR-223. Priming is followed by NLRP3 activation (2), the molecular mechanism of which is not yet fully understood. However, at least one of following three events is commonly required: potassium efflux, cathepsin B release from lysosomes and ROS production. Two direct ROS-related NLRP3 ligands have recently been proposed: TXNIP released from the redox system and oxidized mitochondrial DNA. Mitochondria and mitochondrial ROS seem to play an important role and can be influenced by calcium signalling; whether potassium efflux and lysosomal damage link into mitochondrial signalling as well is yet unknown. Autophagy negatively impacts NLRP3 activation, potentially by removing ROS-producing dysfunctional mitochondria. Furthermore, the second messenger cAMP can bind directly to NLRP3 and inhibit its activation. Extracellular calcium decreases cAMP through CASR signalling. Kinase signalling positively regulates NLRP3 activation through PKR that directly interacts with NLRP3, while Syk kinase activity is required for NLRP3 activation in response to specific ligands such as C. albicans. NLRP3 activation triggers the assembly of active inflammasome complexes containing ASC and pro-caspase-1 (3). Endogenous and viral Pyrinonly proteins (POPs) can interfere with inflammasome assembly, while GBP5 selectively promotes NLRP3 dependent ASC oligomerization. Inflammasome formation results in the processing and activation of caspase-1 (4). Caspase-1 
cleaves the pro-forms of IL-1 $\beta$ and IL-18, releasing the mature cytokines and also induces cell death by pyroptosis.

Figure options

In contrast to oxidative stress, other reports surprisingly found that antioxidants are also essential for inflammasome activation. Macrophages deficient in the antioxidant enzyme SOD1, even though they have increased ROS, have decreased inflammasome activity because of oxidation of redox-sensitive cysteine residues on caspase-1 [18].

These seemingly contradictory findings might be reconciled by a more integrated view of the redox system, where an oxidative insult is always balanced by a cellular antioxidant response and vice versa. This is demonstrated by the fact that TLR stimuli lead to a biphasic redox response, starting off with an oxidative hit and followed by a rapid antioxidant response [19]. In addition, the baseline activation level of the redox system determines the potential for IL-1 $\beta$ production through NLRP3 [20•]. In contrast to primary monocytes, monocytic cell lines or cultured macrophages have a high baseline activation of the antioxidant system, leading to a stunted IL-1 $\beta$ response to NLRP3 stimuli. This highlights the fact that redox dependency is very much determined by the system it is studied in.

The importance of redox signalling could also explain findings that inhibition of autophagy increases IL-1 $\beta$ production by NLRP3 activators. Since dysfunctional organelles, including mitochondria, are removed by autophagosomes, inhibition of autophagy leads to an accumulation of ROS-producing mitochondria, which is accompanied by increased release of mitochondrial DNA [21]. However, autophagy is also involved in the removal of ubiquitinated inflammasomes [22] and pro-IL-1 $\beta$ in activated cells [23].

Regulation of NLRP3 expression by miR-223

Induction of NLRP3 expression by TLR ligands is necessary for optimal inflammasome activation, which is highlighted by the fact that constitutive NLRP3 overexpression is sufficient to induce IL-1 $\beta$ secretion to NLRP3 inflammasome activators [14 and 15••]. Clearly, regulation of NLRP3 levels offers an interesting mechanism to alter the inflammatory potential of immune cells and different cell functions could require a different threshold for NLRP3 activation. We and others recently identified miRNA-223 as a negative regulator

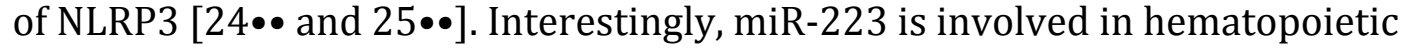
differentiation and differentially expressed among myeloid cells [26]. Thus, it can fine-tune NLRP3 in a cell type-dependent and differentiation status-dependent manner in monocytes, macrophages, dendritic cells and especially granulocytes, which have very high miR-223 expression. Of note, miR-223 deficient mice exhibit phenotypes consistent with deregulated NLRP3, namely neutrophilia, 
spontaneous lung inflammation and increased susceptibility to endotoxin challenge [27].

\section{Viral regulation of NLRP3}

Increasing evidence also suggests that many viruses have evolved mechanisms to dampen inflammasome signalling. Most mechanisms inhibit inflammasome assembly, caspase-1 activation or cytokine neutralization [28]. For example, KSHV ORF63 [29] and Measles virus V protein [30] can bind NLRP3 and prevent its activation. Several poxviruses express viral POPs, which interfere with ASC recruitment, such as shape fibroma virus gp013 [31] and myxoma virus M013 [32]. In addition to the endogenous miR-223, we also identified an EBV miRNA, miR-BART15, that can inhibit NLRP3 through the same target site as miR-223 [24••]. We found that miR-BART15 can be transferred from infected B cells to uninfected cells via exosomes. However, increasing evidence suggests that EBV can also directly infect myeloid cells [33], where it could affect NLRP3 expression. In general, dampening inflammasome signalling could be advantageous to viruses in order to escape pyrogenic effects of IL-1 $\beta$, cell-mediated immunity by IL-18 and cell death of infected cells by pyroptosis.

\section{Ubiquitination as a negative regulator of NLRP3}

Inflammasome-mediated IL-1 $\beta$ production is a two-step process. A primary signal must activate NFKB to initiate pro IL-1 $\beta$ mRNA synthesis followed by a secondary signal that activates the inflammasome and IL-1 $\beta$ release [34]. For the NLRP3 inflammasome it was demonstrated that a primary signal such as TLR activation was also required to prime or 'license' NLRP3 itself. NLRP3 protein expression levels were shown to be a limiting step in inflammasome activation [14 and 35]. Two recent reports have demonstrated that LPS can rapidly prime NLRP3 in a manner distinct from transcriptional induction. In macrophages caspase- 1 activation was shown to occur with simultaneous administration of LPS and the NLRP3 activator ATP, thus bypassing the transcriptional induction of NLRP3 that occurred after two hours of LPS stimulation [15•• and 36]. Juliana et al. further demonstrated that NLRP3 was basally ubiquitinated and that LPS stimulation reduces this ubiquitination in a manner that is dependent on mitochondrial ROS generation and results in NLRP3 activation. Interestingly, ATP could also induce de-ubiquitination of NLRP3 via a ROS-independent mechanism suggesting there are two de-ubiquitinating enzymes that regulate NLRP3 [ $15 \bullet \bullet$. Whether this mechanism is unique to LPS priming of NLRP3 remains to be examined.

\section{A role for protein kinases in NLRP3 activation}

The phosphorylation of NLRC4 on Ser533 mediated by PKC $\delta$ was recently shown to be essential for functional NLRC4 inflammasome formation [37], the first time that an activating covalent modification of any inflammasome has been reported. 
Although phosphorylation of NLRP3 has not been directly demonstrated there is evidence to support a role for kinase signalling in its regulation. A recent study by Lu et al. has demonstrated that RNA-dependent protein kinase (PKR) is a broad inflammasome regulator. Activation of NLRP3, NLRP1, NLRC4 and AIM2 induced PKR phosphorylation and PKR deficiency attenuated caspase-1 activation in response to activation of all of these inflammasomes. Coimmunoprecipitation assays showed that PKR interacts with NLRP3 and furthermore in a cell free system activated PKR together with NLRP3, ASC and caspase-1 reconstituted a functional inflammasome [38•]. Previous work has also demonstrated a role for Syk tyrosine kinase activity in the activation of NLRP3 in response to a number of stimuli. Fungal infection by Candida albicans [39] the Plasmodium metabolite hemozoin [ 40], Mycobacterium tuberculosis infection [41] and carbon nanotubes [42] all activate NLRP3 and require Syk activity for effective inflammasome activation.

\section{Pyrin domain interactions}

NLRP3 has a characteristic tri-domain structure consisting of a C-terminal leucine rich repeat domain, a central nucleotide binding and oligomerization (NACHT) domain and an N-terminal Pyrin domain (PYD) [43]. The PYD domain of NLRP3 is crucial to its function as it interacts with the PYD domain of ASC, which mediates the activation of caspase-1 [2]. Two PYD only proteins (POPs) POP1 and POP2 that negatively regulate NLRP3 inflammasome activation in humans have been described. POP1 (also known as ASC2, ASCI, ASCL and PYDC1) binds to the PYD domain of ASC to which it is 64\% identical and may disrupt the interaction of ASC with other proteins such as NLRP3 [44]. POP2 shows closer homology to the PYD domains of NLRP proteins than that of ASC. POP2 was shown to prevent the recruitment of ASC by NLRP3 [45]. A recent study by Shenoy et al. identified guanylate binding protein 5 (GBP5) as a selective regulator of the NLRP3 inflammasome. GBP5 interacts with the PYD domain of NLRP3 and it was shown that tetramers of GBP5 promote ASC oligomerization through NLRP3. Interestingly, GBP5 only played a role in NLRP3 activation in response live bacterial infection and soluble ligands such as ATP and nigericin but not crystalline substances such as MSU and alum [ 46••]. The crystal structure of the PYD of NLRP3 has been reported [47]. Interestingly, an unexpected disulphide bond between Cys-8 and Cys-108 of the NLRP3 PYD was identified. These Cys residues are evolutionarily conserved, suggesting that redox modifications could influence the structure of NLRP3 PYD [47]. Further structural studies should shed light on the conformational changes in NLRP3 and the mechanism of its activation.

\section{Calcium signalling}

Intracellular Ca2+ store release was previously implicated in NLRP3 dependent IL-1 $\beta$ secretion [48]. A report by Murakami et al. has shown that multiple NLRP3 activators induce Ca2+ signalling. Depletion of endoplasmic reticulum (ER) Ca2+ stores and inhibition of extracellular Ca2+ influx both attenuated ATP stimulated 
NLRP3 activation. Furthermore, pharmacological inhibitors of key Ca2+ signalling mediators such as phospholipase $C$ attenuated IL-1 $\beta$ release. Mechanistically, Ca2+ signalling was found to promote mitochondrial damage and therefore NLRP3 activation [ 49••]. More recently it has been demonstrated that the calcium-sensing receptor (CASR) activates the NLRP3 inflammasome through PLC signalling. In addition it was shown that activation of CASR by extracellular $\mathrm{Ca} 2+$ results in the inhibition of adenylate cyclase and a reduction in cyclic AMP (cAMP) levels. It was found that cAMP binds NLRP3 and negatively

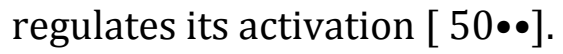

\section{Pharmacological manipulation of inflammasome activity}

The current best treatments for inflammasome disorders target the main product of inflammasome activity, IL-1 $\beta$ [51]. Anti-IL-1 $\beta$ biologicals such as the recombinant IL-1 receptor antagonist Anakinra are clinically successful anti-IL-1 therapies [9•]. Other strategies for treating IL-1 related disease such as developing P2X7 receptor antagonists and caspase- 1 inhibitors have also been explored and tested in clinical trials [9• and 52].

Several previously characterized small-molecule inhibitors have more recently also been shown to affect NLRP3 inflammasome function. Glyburide is a sulfonylurea drug used in the treatment of T2D, where it acts by inhibiting potassium channels in pancreatic $\beta$ cells. Glyburide inhibits IL- $1 \beta$ production in response to multiple NLRP3 stimuli but not NLRC4 or NLRP1 activation. The inhibitory activity of glyburide was not dependent on potassium channels or NLRP3 ATPase activity. Interestingly, glyburide did not inhibit temperatureinduced IL-1 $\beta$ release from monocytes of familial cold autoinflammatory syndrome (FCAS) patients, suggesting it does not directly inhibit NLRP3 but upstream signalling [53•]. We have also found that a compound termed CRID3 can act on or close to NLRP3 and block IL-1 $\beta$ production [54].

Parthenolide is a sesquiterpene lactone that has multiple anti-inflammatory properties. Separate to its effects on NFKB activation parthenolide has now also been shown to inhibit caspase- 1 and NLRP3. Parthenolide inhibited caspase-1 activation in response to NLRP3, NLRP1 and NLRC4 stimulation. The authors suggest this is a result of alkylation of caspase-1 on a number of Cys residues. Parthenolide also directly inhibits NLRP3 by inhibiting its ATPase activity that is required for activation [55]. Bay 11-7082 is another NFKB inhibitor that was also found to specifically inhibit NLRP3. Bay 11-7082 inhibits the ATPase activity of NLRP3 suggesting that this may be the mechanism of inhibition [55].

Currently available inhibitors of inflammasome function have either not been clinically successful or have multiple targets. The development of small molecule 
inhibitors that directly target the NLRP3 inflammasome could provide a cheaper and less invasive therapy for IL-1 $\beta$ and inflammasome-related diseases.

\section{Conclusions}

Our understanding of the precise biochemical control of the NLRP3 inflammasome is still sparse. We still have no clear mechanism for NLRP3 activation by ROS. However the recent insights into control by miR-223, viral manipulation, role of protein kinases and calcium represent an excellent start in the effort to understand how NLRP3 is controlled. Given the importance of NLRP3 for inflammatory diseases, more information on its modulation will help our understanding of NLRP3 in disease and its potential manipulation therapeutically. 
1. Wen $H$, Ting JP, O'Neill LA: A role for the NLRP3 inflammasome in metabolic diseases - did Warburg miss inflammation? Nat Immunol 2012, 13:352-357.

2. Agostini L, Martinon F, Burns K, McDermott MF, Hawkins PN, Tschopp J: NALP3 forms an IL-1beta-processing inflammasome with increased activity in Muckle-Wells autoinflammatory disorder. Immunity 2004, 20:319-325.

3. Martinon F, Burns K, Tschopp J: The inflammasome: a molecular platform triggering activation of inflammatory caspases and processing of prolL-beta. $\mathrm{Mol}$ Cell 2002, 10:417-426.

4. Tschopp J, Schroder K: NLRP3 inflammasome activation: the convergence of multiple signalling pathways on ROS production? Nat Rev Immunol 2010, 10:210-215.

5. Martinon F, Petrilli V, Mayor A, Tardivel A, Tschopp J: Goutassociated uric acid crystals activate the NALP3 inflammasome. Nature 2006, 440:237-241.

6. Masters SL, Dunne A, Subramanian SL, Hull RL, Tannahill GM, Sharp FA, Becker C, Franchi L, Yoshihara E, Chen Z et al.: Activation of the NLRP3 inflammasome by islet amyloid polypeptide provides a mechanism for enhanced IL-1beta in type 2 diabetes. Nat Immunol 2010, 11:897-904.

7. Duewell P, Kono H, Rayner KJ, Sirois CM, Vladimer G, Bauernfeind FG, Abela GS, Franchi L, Nunez G, Schnurr M et al: NLRP3 inflammasomes are required for atherogenesis and activated by cholesterol crystals. Nature 2010, 464:1357-1361.

8. Neven B, Prieur AM, Quartier dit Maire P: Cryopyrinopathies: update on pathogenesis and treatment. Nat Clin Pract Rheumatol 2008, 4:481-489.

9. Dinarello CA, Simon A, van der Meer JW: Treating inflammation

- by blocking interleukin-1 in a broad spectrum of diseases. Nat Rev Drug Discov 2012, 11:633-652.

Nice review of the role of IL-1 $1 \beta$ in the pathogenesis of numerous human diseases.

10. Dostert C, Petrilli V, Van Bruggen R, Steele C, Mossman BT, Tschopp J: Innate immune activation through Nalp3 inflammasome sensing of asbestos and silica. Science 2008, 320:674-677. 
11. van Bruggen $R$, Koker MY, Jansen M, van Houdt M, Roos D, Kuijpers TW, van den Berg TK: Human NLRP3 inflammasome activation is Nox1-4 independent. Blood 2010, 115:5398-5400.

12. Homung V, Bauernfeind F, Halle A, Samstad EO, Kono H, Rock KL, Fitzgerald KA, Latz E: Silica crystals and aluminum salts activate the NALP3 inflammasome through phagosomal destabilization. Nat Immunol 2008, 9:847-856.

13. Zhou R, Yazdi AS, Menu P, Tschopp J: A role for mitochondria in

- NLRP3 inflammasome activation. Nature 2011, 469:221-225.

This study provides convincing evidence for a central role of mitochondria and mtROS in NLRP3 inflammasome activation.

14. Bauernfeind FG, Horvath G, Stutz A, Alnemri ES, MacDonald K, Speert D, Fernandes-Alnemri T, WuJ, Monks BG, Fitzgerald KA et af: Cutting edge: NF-kappaB activating pattern recognition and cytokine receptors license NLRP3 inflammasome activation by regulating NLRP3 expression. J Immuno/ 2009, 183:787-791.

15. Juliana C, Fernandes-Alnemri T, Kang S, Farias A, Qin F,

-. Alnemri ES: Non-transcriptional priming and deubiquitination regulate NLRP3 inflammasome activation. $J$ Biol Chem 2012, 287:36617-36622.

This report provides first evidence of inhibitory ubiquitination of NLRP3. Functionally, de-ubiquitination is involved in priming and activation of the NLRP3 inflammasome.

16. Zhou R, Tardivel A, Thorens B, Choi I, Tschopp J: Thioredoxininteracting protein links oxidative stress to inflammasome activation. Nat Immunol 2010, 11:136-140.

17. Shimada K, Crother TR, Karlin J, Dagvadorj J, Chiba N, Chen S,

-. Ramanujan VK, Wolf AJ, Vergnes L, Ojcius DM ot al.: Oxidized mitochondrial DNA activates the NLRP3 inflammasome during apoptosis. Immunity 2012, 36:401-414.

These findings suggest a unified model of NLRP3 activation with mitochondrial homeostasis at the centre and oxidized mtDNA as a direct NLRP3 ligand. This also suggests a close link between apoptosis and the inflammasome at the mitochondrial level.

18. Meissner F, Molawi K, Zychlinsky A: Superoxide dismutase 1 regulates caspase-1 and endotoxic shock. Nat lmmunol 2008, 9:866-872.

19. Tassi S, Carta S, Vene R, Delfino L, Ciriolo MR, Rubartelli A: Pathogen-induced interleukin-1beta processing and secretion is regulated by a biphasic redox response. $J$ immunol 2009, 183:1456-1462.

20. Carta S, Tassi S, Pettinati I, Delfino L, Dinarello CA, Rubartelli A:

- The rate of interleukin-1beta secretion in different myeloid cells varies with the extent of redox response to Toll-like receptor triggering. J Biol Chem 2011, 286:27069-27080.

This and other publications by the Rubartelli group highlight the importance of the cellular redox status for NLRP3 activation. In particular, cells with basally activated antioxidant signalling cannot produce IL-1 $\beta$ efficiently upon NLRP3 activation.

21. Nakahira K, Haspel JA, Rathinam VA, Lee SJ, Dolinay T, Lam HC, Englert JA, Rabinovitch M, Cernadas M, Kim HP et al.: Autophagy proteins regulate innate immune responses by inhibiting the release of mitochondrial DNA mediated by the NALP3 inflammasome. Nat Immunol 2011, 12:222-230.

22. Shi CS, Shenderov K, Huang NN, Kabat J, Abu-Asab M, Fitzgerald KA, Sher A, Kehrl JH: Activation of autophagy by inflammatory signals limits IL-1beta production by targeting ubiquitinated inflammasomes for destruction. Nat immunol 2012, 13:255-263.

23. Harris J, Hartman M, Roche C, Zeng SG, O'Shea A, Sharp FA, Lambe EM, Creagh EM, Golenbock DT, Tschopp J et al.: Autophagy controls IL-1beta secretion by targeting pro-IL1 beta for degradation. J Bial Chem 2011, 286:9587-9597.

24. Haneklaus M, Gerlic M, Kurowska-Stolarska M, Rainey AA, Pich D,

-. Mclnnes IB, Hammerschmidt W, O'Neill LA, Masters SL: Cutting edge: miR-223 and EBV miR-BART15 regulate the NLRP3 inflammasome and IL-1beta production. $J /$ mmunol 2012 , 189:3795-3799.

See Ref. [25].

25. Bauernfeind F, Rieger A, Schildberg FA, Knolle PA, Schmid-

•. BurgkJL, Hornung V: NLRP3 inflammasome activity is negatively controlled by miR-223. $J$ /mmunol 2012, 189:4175-4181. 
26. Chen CZ, Li L, Lodish HF, Bartel DP: MicroRNAs modulate hematopoietic lineage differentiation. Science 2004, 303:83-86.

27. Johnnidis JB, Harris MH, Wheeler RT, Stehling-Sun S, Lam MH, Kirak O, Brummelkamp TR, Fleming MD, Camargo FD: Regulation of progenitor cell proliferation and granulocyte function by microRNA-223. Nature 200B, 451:1125-1129.

28. Gram AM, Frenkel J, Ressing ME: Inflammasomes and viruses: cellular defence versus viral offence. J Gen Viral 2012, 93-2063-2075.

29. Gregory SM, Davis BK, West JA, Taxman DJ, Matsuzawa S, Reed JC, Ting JP, Damania B: Discovery of a viral NLR homolog that inhibits the inflammasome. Science 2011, 331:330-334.

30. Komune $\mathrm{N}$, Ichinohe $\mathrm{T}$, Ito $\mathrm{M}$, Yanagi $\mathrm{Y}$ : Measles virus $\mathbf{V}$ protein inhibits NLRP3 inflammasome-mediated interleukin-1beta secretion. J Virol 2011, 85:13019-13026.

31. Dorfleutner A, Talbott SJ, Bryan NB, Funya KN, Rellick SL, Reed JC, Shi X, Rojanasakul Y, Flynn DC, Stehlik C: A Shope Fibroma virus PYRIN-only protein modulates the host immune response. Virus Genes 2007, 35:685-694.

32. Johnston JB, Barrett JW, Nazarian SH, Goodwin M, Ricciuto D, Wang G, McFadden G: A poxvirus-encoded pyrin domain protein interacts with ASC-1 to inhibit host inflammatory and apoptotic responses to infection. Immunity 2005, 23:587-598.

33. Savard M, Belanger C, Tardif M, Gourde P, Flamand L, Gosselin J: Infection of primary human monocytes by Epstein-Barr virus. $J$ Virol 2000, 74-2612-2619.

34. Bauernfeind F, Ablasser A, Bartok E, Kim S, Schmid-Burgk J, Cavlar T, Hornung V: Inflammasomes: current understanding and open questions. Cell Mol Life Sci 2011, 68:765-783.

35. Franchi L, Eigenbrod T, Nunez G: Cutting edge: TNF-alpha mediates sensitization to ATP and silica via the NLRP3 inflammasome in the absence of microbial stimulation. $J$ Immunol 2009, 183:792-796.

36. Schroder K, Sagulenko V, Zamoshnikova A, Richards AA, Cridland JA, Irvine KM, Stacey KJ, Sweet MJ: Acute lipopolysaccharide priming boosts inflammasome activation independently of inflammasome sensor induction. immunobiology 2012, 217:1325-1329.

37. Qu Y, Misaghi S, Izrael-Tomasevic A, Newton K, Gilmour LL, Lamkanfi M, Louie S, Kayagaki N, Liu J, Komuves L et al.: Phosphorylation of NLRC4 is critical for inflammasome activation. Nature 2012, 490:539-542.

38. Lu B, Nakamura T, Inouye K, Li J, Tang Y, Lundback P, Valdes-

- Ferrer SI, Olofsson PS, Kalb T, Roth J et al.: Novel role of PKR in inflammasome activation and HMGB1 release. Nature 2012, 488:670-674.

Demonstrates that PKR is a positive regulator of multiple inflammasomes. It also shows a direct interaction between NLRP3 and PKR, which clearly implicates that kinase signalling plays a role in the activation of NLRP3.

39. Gross O, Poeck H, Bscheider M, Dostert C, Hannesschlager N, Endres S, Hartmann G, Tardivel A, Schweighoffer E, Tybulewicz V et al.: Syk kinase signalling couples to the Nlrp3 inflammasome for anti-fungal host defence. Nature 2009, 459:433-436.

40. Shio MT, Eisenbarth SC, Savaria M, Vinet AF, Bellemare MJ, Harder KW, Sutterwala FS, Bohle DS, Descoteaux A, Flavell RA et al.: Malarial hemozoin activates the NLRP3 inflammasome through Lyn and Syk kinases. PLoS Pathog 2009, 5:e1000559. 
41. Wong KW, Jacobs WR Jr: Critical role for NLRP3 in necrotic death triggered by Mycobacterium tuberculosis. Cell Microbiol 2011, 13:1371-1384.

42. Palomaki J, Valimaki E, Sund J, Vippola M, Clausen PA, Jensen KA, Savolainen K, Matikainen S, Alenius H: Long, needlelike carbon nanotubes and asbestos activate the NLRP3 inflammasome through a similar mechanism. ACS Nano 2011, 5:6861-6870.

43. Bryant C, Fitzgerald KA: Molecular mechanisms involved in inflammasome activation. Trends Cell Biol 2009, 19:455-464.

44. Stehlik C, Krajewska M, Welsh K, Krajewski S, Godzik A, Reed JC: The PAAD/PYRIN-only protein POP1/ASC2 is a modulator of ASC-mediated nuclear-factor-kappa B and pro-caspase-1 regulation. Biochem J 2003, 373:101-113.

45. Bedoya F, Sandler LL, Harton JA: Pyrin-only protein 2 modulates NF-kappaB and disrupts ASC:CLR interactions. $J / m m u n o l$ 2007, 178:3837-3845.

46. Shenoy AR, Wellington DA, Kumar P, Kassa $\mathrm{H}$, Booth $\mathrm{CJ}$,

-. Cresswell P, MacMicking JD: GBP5 promotes NLRP3 inflammasome assembly and immunity in mammals. Science 2012, 336:481-485.

Shows that a non-NLR protein, GBP5 promotes NLRP3 inflammasome assembly through interaction with the pyrin domain. Interestingly, GBP5 is only required for responses to certain NLRP3 stimuli.

47. Bae JY, Park HH: Crystal structure of NALP3 protein pyrin domain (PYD) and its implications in inflammasome assembly. $J$ Biol Chem 2011, 286:39528-39536.

48. Brough D, Le Feuvre RA, Wheeler RD, Solovyova N, Hifiker S, Rothwell NJ, Verkhratsky A: $\mathrm{Ca}^{2+}$ stores and $\mathrm{Ca}^{2+}$ entry differentially contribute to the release of IL-1 beta and IL-1 alpha from murine macrophages. J Immunal 2003, 170:3029-3036.

49. Murakami T, Ockinger J, Yu J, Byles V, McColl A, Hofer AM,

-. Horng T: Critical role for calcium mobilization in activation of the NLRP3 inflammasome. Proc Natj Acad Sci USA 2012, 109:11282-11287.

This report shows that multiple NLRP3 stimuli activate intracellular $\mathrm{Ca}^{2+} \mathrm{r}$ elease and that inhibition of $\mathrm{Ca}^{2+}$ signalling attenuates NLRP3 activation.

50. Lee GS, Subramanian N, Kim Al, Aksentijevich I, Goldbach-

-. Mansky R, Sacks DB, Germain RN, Kastner DL, Chae JJ: The calcium-sensing receptor regulates the NLRP3 inflammasome through $\mathrm{Ca}(2+)$ and cAMP. Nature 2012 , 492:123-127.

Demonstrates that high extracellular $\mathrm{Ca}^{2+}$ activates NLPP3 through CASR and that CAMP is a negative regulator of NLRP3.

51. Lopez-Castejon G, Pelegrin P: Current status of inflammasome blockers as anti-inflammatory drugs. Expert Opin Imvestig Drugs 2012, 21:995-1007.

52. Arulkumaran N, Unwin RJ, Tam FW: A potential therapeutic role for P2X7 receptor (P2X7R) antagonists in the treatment of inflammatory diseases. Expert Opin imvestig Drugs 2011, 20:897-915.

53. Lamkanfi M, Mueller JL, Vitari AC, Misaghi S, Fedorova A

- Deshayes K, Lee WP, Hoffman HM, Dixit VM: Glyburide inhibits the cryopyrin/Nalp3 inflammasome. J Cell Biol 2009, 187:61-70.

Clearly demonstrates that the T2D treatment glyburide is also a specific inhibitor of NLRP3 mediated IL-1 $\beta$ secretion.

54. Coll RC, O'Neill LA: The cytokine release inhibitory drug CRID3 targets ASC oligomerisation in the NLRP3 and AIM2 inflammasomes. PLoS One 2011, 6:e29539.

55. Juliana C, Fernandes-Alnemri T, Wu J, Datta P, Solorzano L, Yu JW, Meng R, Quong AA, Latz E, Scott CP et al.: Antiinflammatory compounds parthenolide and Bay 11-7082 are direct inhibitors of the inflammasome. $J$ Bial Chem 2010, 285:9792-9802. 